\title{
Efficient Spectral-Galerkin Method for eigenvalue problems
}

\author{
Zhang Jun \\ Guizhou University of Finance and Econmics \\ School of Mathematics and Statistical \\ Guiyang, 550025, China. \\ e-mail:zj654440@163.com
}

\author{
Fan xinyue \\ School of Faculty of Science \\ Guizhou University \\ Guiyang, 550025, China. \\ e-mail:fan.xinyue@163.com
}

\begin{abstract}
-we provide a priori error estimates for linear elliptic eigenvalue problems based on the spectral-Galerkin method, and also provide an efficient Galerkin method is proposed for solving this problems, with this scheme, the solution of an eigenvalue problem on a big spectral space is reduced to the solution of an eigenvalue problem on a small spectral space and and solution of a linear algebraic system on the big spectral space and the resulting solution still maintains an asymptotically optimal accury.
\end{abstract}

Keywords-eigenvalue; iterated galerkin method; priori error estimates; legendre polynomia

\section{INTRODUCTION}

The purpose of this paper is to present an efficient technique based on thLegendre-Galkerkin approximations for solving eigenvalue problems, and also provide a priori error estimates for linear elliptic eigenvalue problems based on the spectral-Galerkin method that different from [1]. It should be mentioned that the this method is called iterated Galerkin method. This method for elliptic eigenvalue problems is first proposed in [2], and later developed in [3], then it was further nvestigated by many other authors, for instance Axelsson and Layton [4] for nonlinear elliptic problems, Dawson and Wheeler [5] and $\mathrm{Xu}[6]$ for finite difference scheme for parabolic equations, Layton and Lenferink [7], and Utnes[8] for Navier-Stokes equations Marion and $\mathrm{Xu}[9]$ for evolution equations. But we do not see is was used in spectral spaces.

Inspired by the work [10], in this paper we propose iterated Galerkin method for eigenvalue problems. By using the scheme, the solution of an eigenvalue problem on a big big spectral space is reduced to the solution of an eigenvalue problem on a small spectral space and solution of a linear algebraic system on the big spectral space,it provides very accurate approximations with a relatively small number of unknowns, because solving an elliptic eigenvalue problem will not by much more difficult than the solution of some standard elliptic boundary value problem.

In the remainder of this section, we would like to give a example to illustrate the main idea in this paper. Consider the following eigenvalue problem

$$
-\Delta u=\lambda u, \text { in } \Omega, u=0, \text { on } \partial \Omega
$$

the variational problem associated with (1) is given by: find

This work partially supported by Foundation of Guizhou Science and Technology Department (Grant No.[2013]2128) $\lambda \in R$ and $u \in H_{0}^{1}(\Omega)$ with $\|u\|_{b}=1$ satisfying:

$$
a(u, v)=\lambda b(u, v), \quad \forall v \in H_{0}^{1}(\Omega),
$$

where

$$
\begin{gathered}
a(u, v)=\int_{\Omega} \nabla u \nabla v, \\
b(u, v)=\int_{\Omega} u v,\|b\|_{b}=b(u, u)^{\frac{1}{2}} .
\end{gathered}
$$

Let us first introduce some bsic notations which will be used in the sequel.We assume that $\Omega=(-1,1)^{d}, d=1,2,3$, we denote by $\operatorname{Ln}(\mathrm{x})$ the $\mathrm{nth}$ degree Legendre polynomial, and set

$$
\begin{aligned}
& S_{N}=\operatorname{span}\left\{L_{0}(x), L_{1}(x), \cdots, L_{N}(x)\right\}, \\
& X_{N}=\left\{v \in S_{N}^{d}:\left.v\right|_{\partial \Omega}=0\right\} .
\end{aligned}
$$

Then the standard Legendre -Galerkin approximation to (2) is: find $\lambda_{N} \in R$ and $u_{N} \in X_{N}$ with $\left\|u_{N}\right\|_{b}=1$ such that

$$
a\left(u_{N}, v\right)=\lambda_{N} b\left(u_{N}, v\right), \quad \forall v \in X_{N} \text {. }
$$

We can employ the following high order algorithm to approximate the problem (1), say the first eigenvalue $\lambda$ with its corresponding eigenvector $u$ and $\left\|u_{N}\right\|_{b}=1$ :

Step1: solve an eigenvalue problem on a small spectral space: find $\lambda_{N} \in R$ and $u_{N} \in X_{N}$ with such that $\left\|u_{N}\right\|_{b}=1$ and

$$
a\left(u_{N}, v\right)=\lambda_{N} b\left(u_{N}, v\right), \quad \forall v \in X_{N} .
$$

Step 2: solve one singe linear problem on a big spectral space: find $u_{\square}^{*} \in X_{\square}$ such that

$$
a\left(u_{\square}^{*}, v\right)=\lambda \downarrow b\left(u_{\square}, v\right), \quad \forall v \in X_{\square} .
$$

Step 3: compute the Rayleigh quotient

$$
\lambda_{\square}^{*}=\frac{a\left(u_{\square}^{*}, u_{\square}^{*}\right)}{\left(u_{\square}^{*}, u_{\square}^{*}\right)} .
$$

if $u \in H^{s}, s>1$ then we can establish the following results(see sections 4$)$

$$
\begin{gathered}
\left\|u_{\square}^{*}-u\right\|_{a}=O\left(N^{1-s}+N^{-s}\right), \\
\left|\lambda_{\square}^{*}-\lambda\right|=O\left(\square^{2-2 s}+N^{-2 s}\right) .
\end{gathered}
$$




\section{PRELIMINARIES}

In this scetion, we shall describe some basic notation and properties of the Legendre-Galerkin approximation. Throughoutout this paper, we shall use the letter $C$ (with or without subscripts) to denote a generic positive constant which may stand for different values at different occurrences.

Suppose that $H$ is a real Hilbert space with inner product $(\cdot, \cdot)_{H}$ and norm $\|\cdot\|_{H}$. Let $a(\cdot, \cdot)$,

$b(\cdot, \cdot)$ be two symmetric bilinear forms on $H \times H$

Satisfying

$a(w, v) \leq C\|w\|_{H}\|v\|_{H}, \forall w, v \in H,\|w\|_{H}^{2} \leq C a(w, w), \forall w \in H$, $|b(u, v)| \leq C\|u\|_{H}\|v\|_{H}, 0<b(w, w), \forall u, v, w, \in H$.

We note that $\|\cdot\|_{a} \equiv a(\cdot, \cdot)^{\frac{1}{2}}$ and $\|\cdot\|_{H}$ are two equivalent norms on $H$, we assume that the norm $\|\cdot\|_{H}$ is relatively compact with respect to the norm $\|w\|_{a} \equiv b(w, w)^{\frac{1}{2}}$. In the sense the form any sequence which is bounded in $\|\cdot\|_{H}$ one can extract a subsequence which is Canchy with respect to $\|\cdot\|_{b}$. In the rest of this paper we shall use $a(\cdot, \cdot)$ and $\|\cdot\|_{a}$ as the inner product and norm on $H$. Consider the source problem (4) associated with (1) and the approximate sourec problem(5) associated with (3): find $u \in H_{0}^{1}(\Omega)$ such that

$$
a(u, v)=b(f, v), \quad \forall v \in H_{0}^{1}(\Omega),
$$

find $u_{N} \in X_{N}$ such that

$$
a(u, v)=b(f, v), \quad \forall v \in X_{N},
$$

At first, we transform (4)-(5)into the operator forms, note that $a(\cdot, \cdot)$ is coercive. Using the source problem (4) associated with (2), we define the operator

$$
\begin{aligned}
T: L^{2}(\Omega) & \rightarrow H_{0}^{1}(\Omega), \\
a(T u, v) & =b(f, v), \quad \forall v \in H_{0}^{1}(\Omega),
\end{aligned}
$$

Babuska and Osborn [1] proved that (2) has the operator form:

$$
T u=\frac{1}{\lambda} u
$$

Using the source problem (5) associated with (3), we define the operator $T_{N}: L^{2}(\Omega) \rightarrow X_{N}$

$$
a\left(T_{N} u, v\right)=b(f, v), \quad \forall v \in X_{N},
$$

Bramble and Osborn [1] also proved that (3) has the operator form:

$$
T_{N} u_{N}=\frac{1}{\lambda_{N}} u_{N}
$$

Next, we prove that $T$ and $T_{N}$ are self-adjoint operators and $\left\|T_{N}-T\right\|_{b} \rightarrow 0$ as $N \rightarrow \infty$. Since $\forall f, g \in L^{2}(\Omega)$

$$
b(T f, v)=b(g, T f)=a(T g, T f)=a(T f, T g)=b(f, T g)
$$

$T$ is is a self-adjoint operator. In a similar way we can also prove $T_{N}$ is a self-adjoint operator.

It is well known(cf.[3]) that for $s \geq 1$ and $u \in H^{s}(\Omega)$, the following optimal error hold:

$$
\left\|u-u_{N}\right\|_{b}+N^{-1}\left\|u-u_{N}\right\|_{a} \leq C(s) N^{-s}\|u\|_{s} .
$$

Hence we have

$$
\left\|T_{N}-T\right\|_{b}=\sup _{g \in L^{2}(\Omega)} \frac{\left\|T_{N} g-T g\right\|_{b}}{\|g\|_{b}} \leq C N^{-s},
$$

This show that $\left\|T_{N}-T\right\|_{b} \rightarrow 0$ as $N \rightarrow \infty$.

\section{A PRIORI ERROR ESTIMATES OF SPECTRAL-GALERKIN METHOD SELECTING A TEMPLATE}

In this section we will provide a priori error estimates for linear elliptic eigenvalue problems based on the spectralGalerkin method that different from[1]. It is known that (2) has a countable sequence of real eigenvalues $0 \leq \lambda_{1} \leq \lambda_{2} \leq \lambda_{3} \leq \cdots$, and corresponding eigenvectors $u_{1}, u_{2}, u_{3}, \cdots$, which can be assumesd to satisfy

$$
a\left(u_{i}, u_{j}\right)=\lambda_{j} b\left(u_{i}, u_{j}\right)=\delta_{i j}
$$

In the sequence $\left\{\lambda_{j}\right\}$, the $\lambda_{j}$ are repeated according to geometric multiplicity. It is known that (3) has a finite sequence of eigenvalues $0 \leq \lambda_{1, N} \leq \lambda_{2, N} \leq \lambda_{3, N} \leq \cdots$, and corresponding eigenvectors $u_{1, N}, u_{2, N}, u_{3, N}, \cdots$, which can be assumesd to satisfy $a\left(u_{i, N}, u_{j, N}\right)=\lambda_{j, N} b\left(u_{i, N}, u_{j, N}\right)=\delta_{i j}$.

Set $M(\lambda)$ be the space spanned by eigenfunctions of $T$ corresponding to $\lambda$. Let $f=\lambda_{N} u_{N}$ in (4), where $\left(\lambda_{N}, u_{N}\right)$ is an eigenvalue pair of (2), a special solution of (2) is : find $u^{o}$ such that

$$
a\left(T_{N} u, v\right)=\lambda_{N}\left(u_{N}, v\right), \forall v \in H_{0}^{1}(\Omega) .
$$

Lemma 3.1 Assume $\left(\lambda_{N}, u_{N}\right)$ ais an eigenvalue pair of (3), $\|\cdot\|_{b}=1, \lambda_{N} \rightarrow \lambda$ as $N \rightarrow \infty$. Let $E$ is the orthogonal- 
projection operator from $L^{2}(\Omega)$ onto $M(\lambda)$, taking $u=\frac{E u_{N}}{\left\|E u_{N}\right\|_{b}}$.

Then

$$
\begin{aligned}
& \frac{\lambda_{N}-\lambda}{\lambda}=\frac{1}{b\left(u, u_{N}\right)} b\left(u^{o}-u_{N}, u\right), \\
& \left\|u_{N}-u\right\|_{b} \leq \lambda_{N}^{-1} \frac{\left\|u_{N}-u\right\|_{b}}{d\left(\lambda^{-1}\right)}\left(1+\frac{1}{\lambda_{N}^{2}} \frac{\left\|u_{N}-u\right\|_{b}^{2}}{d\left(\lambda^{-1}\right)}\right)^{\frac{1}{2}} .
\end{aligned}
$$

where $d\left(\lambda^{-1}\right)$ is disjunctive constant (see [1]),

$$
d(\lambda)=\min _{\lambda_{j} \neq \lambda}\left|\lambda_{N}^{-1}-\lambda_{j}^{-1}\right| \text {. }
$$

Proof. Assume $(\lambda, u)$ is an eigenvalue pair of (3), then

$$
\begin{aligned}
& b\left(u^{o}-u_{N}, u\right)=b\left(u^{o}, u\right)-\left(u_{N}, u\right) \\
& =\lambda^{-1} a\left(u^{o}, u\right)-b\left(u_{N}, u\right)=\lambda^{-1} \lambda_{N} b\left(u_{N}, u\right)-b\left(u_{N}, u\right) \\
& =\frac{\lambda_{N}-\lambda}{\lambda} b\left(u_{N}, u\right) .
\end{aligned}
$$

We obtain (7). Denote $\left\{\lambda_{j}\right\}$ be the set of all eigenvalue, and $u_{j}$ be the eigenfunction corresponding to $\lambda_{j},\left\{u_{j}\right\}$

Construct complete orthogonal system, then

$$
\begin{aligned}
& u_{N}=\sum_{j} b\left(u_{N}, u_{j}\right) u_{j}, \\
& T u_{N}=\sum_{j} \lambda_{N}^{-1} b\left(u_{N}, u_{j}\right) u_{j} .
\end{aligned}
$$

Because $\mathrm{E}$ be the orthogonal-projection, we have

$$
E u_{N}=\sum_{\lambda_{j}=\lambda} b\left(u_{N}, u_{j}\right) u_{j} .
$$

Combining (9)-(10)and the define of $u$, we have

$$
\begin{aligned}
& \frac{1}{b\left(u_{N}, u\right)}-1=\frac{1-b\left(u_{N}, u\right)}{b\left(u_{N}, u\right)}=\frac{1}{2} \frac{\left\|u_{N}, u\right\|_{b}^{2}}{b\left(u_{N}, u\right)}, \\
& b\left(u_{N}, u\right)=\frac{b\left(\sum_{\lambda_{j}=\lambda} b\left(u_{N}, u_{N}\right) u_{j}, \sum_{j} b\left(u_{N}, u_{N}\right) u_{j}\right)}{\left\|E u_{N}\right\|_{b}}=\left\|E u_{N}\right\|_{b}, \\
& b\left(u_{N}, E u_{N}\right)=b\left(\sum_{j} b\left(u_{N}, u_{j}\right) u_{j}, \sum_{\lambda_{j}=\lambda} b\left(u_{N}, u_{j}\right) u_{j}\right)=\left\|E u_{N}\right\|_{b},
\end{aligned}
$$

$$
\begin{aligned}
& \left\|E u_{N}-u_{N}\right\|_{b}^{2}=\sum_{\lambda_{j} \neq \lambda} b\left(u_{N}, u_{j}\right)^{2} \leq \sum_{\lambda_{j} \neq \lambda} \frac{\left(\lambda_{N}^{-1}-\lambda_{j}^{-1}\right) b\left(u_{N}, u_{j}\right)^{2}}{d\left(\lambda^{-1}\right)} \\
& =\sum_{\lambda_{j} \neq \lambda} \frac{\left(\lambda_{N}^{-1} b\left(u_{N}-u_{j}\right) u_{j}-\lambda_{j}^{-1} b\left(u_{N}-u_{j}\right) u_{j}\right)^{2}}{d\left(\lambda^{-1}\right)^{2}}=\frac{\left\|T_{N} u_{N}-T u_{N}\right\|_{b}^{2}}{d\left(\lambda^{-1}\right)^{2}} .
\end{aligned}
$$

Then

$$
\begin{aligned}
& \left\|u_{N}-u\right\|_{b}^{2}=2-2 b\left(u_{N}, u\right)=2-2\left\|E u_{N}\right\|_{b} \\
& =2\left(1-\sqrt{1-\left\|u_{N}-E u_{N}\right\|_{b}^{2}}\right),
\end{aligned}
$$

and use inequality

$$
\sqrt{1-a} \geq 1-\frac{1}{2} a(a+1), \forall a \in R,|a|<1 .
$$

We have

$$
\begin{gathered}
\left\|u_{N}-u\right\|_{b}^{2} \leq\left\|u_{N}-E u_{N}\right\|_{b}^{2}\left(1+\left\|u_{N}-E u_{N}\right\|_{b}^{2}\right) \\
\leq \frac{\left\|T_{N} u_{N}-T u_{N}\right\|_{b}^{2}}{d\left(\lambda^{-1}\right)^{2}}\left(1+\frac{\left\|T_{N} u_{N}-T u_{N}\right\|_{b}^{2}}{d\left(\lambda^{-1}\right)^{2}}\right) .
\end{gathered}
$$

Then

$\left\|u_{N}-u\right\|_{b}^{2} \leq \frac{\left\|T_{N} u_{N}-T u_{N}\right\|_{b}}{d\left(\lambda^{-1}\right)^{2}}\left(1+\frac{\left\|T_{N} u_{N}-T u_{N}\right\|_{b}^{2}}{d\left(\lambda^{-1}\right)^{2}}\right)^{\frac{1}{2}}$.

By the define of $T$ and $T_{N}$, we have

$$
u^{o}=T \lambda_{N} u_{N}, u_{N}=T_{N} \lambda_{N} u_{N} .
$$

Combining with (11), (12) we obtain (8). This completes the proof.

Theorem 3.1 Let $\left(\lambda_{N}, u_{N}\right)$ be an eigenvalue pair of (3), $(\lambda, u)$ is an eigenvalue pair of (2) $\lambda_{N} \rightarrow \lambda$ as $N \rightarrow \infty$, $u \in H_{0}^{1}(\Omega), s>1$. Then

$$
\begin{aligned}
& \left\|u_{N}-u\right\|_{b} \leq C(\lambda) C(s) N^{-s}, \\
& \left\|u_{N}-u\right\|_{a} \leq C(\lambda) C(s) N^{-s}, \\
& \left|\lambda_{N}-\lambda\right| \leq C(\lambda) C(s) N^{-s},
\end{aligned}
$$

Proof. Let $f=\lambda_{N} u_{N}$ in (4), where $\left(\lambda_{N}, u_{N}\right)$ is an eigenvalue pair of (3), let $u^{o}$ be an exect solution solution of (4). Obviously, $u_{N}$ be an approximate solution. Because of (6) we have

$$
\begin{aligned}
& \left\|u_{N}-u^{o}\right\|_{b} \leq C(\lambda) C(s) N^{-s}, \\
& \left\|u_{N}-u^{o}\right\|_{a} \leq C(\lambda) C(s) N^{-s},
\end{aligned}
$$

Submit (16) to Leamma 3.1, we obtain (13), and (15). Let $f=\lambda u$ in (4), where $(\lambda, u)$ is an eigenvalue pair of (2), 
$\bar{u}_{N}$ be an approximate solution. That is find $\bar{u}_{N} \in X_{N}$, such that

$$
a\left(\bar{u}_{N}, v\right)=\lambda_{N}\left(u_{N}, v\right), \forall v \in X_{N} .
$$

Because of (6) we have

Because of (6) we have

$$
\begin{aligned}
& \left\|\bar{u}_{N}-u\right\|_{b} \leq C(s) N^{-s}\|u\|_{s}, \\
& \left\|\bar{u}_{N}-u\right\|_{a} \leq C(s) N^{1-s}\|u\|_{s} .
\end{aligned}
$$

Combining with (3), (17), we have

$$
a\left(u_{N}-\bar{u}_{N}, v\right)=b\left(\lambda_{N} u_{N}-\lambda u, v\right), \forall v \in X_{N} .
$$

Take $v=u_{N}-\bar{u}_{N}$, we obtain

$$
\left\|u_{N}-\bar{u}_{N}\right\|_{a}^{2} \leq C\left\|\lambda_{N} u_{N}-\lambda u\right\|_{b}\left\|u_{N}-\bar{u}_{N}\right\|_{b} .
$$

Then we have

$$
\left\|u_{N}-\bar{u}_{N}\right\|_{a} \leq C\left(\left\|\lambda_{N} u_{N}-\lambda u\right\|_{b}+\left\|u_{N}-\bar{u}_{N}\right\|_{b}\right) .
$$

By using triangle inequality we obtain

$$
\begin{aligned}
& \left\|u-u_{N}\right\|_{a} \leq\left\|u-\bar{u}_{N}\right\|_{a} \\
& +C\left(\left|\lambda_{N}-\lambda\|\| u_{N}\left\|_{b} \mid+\lambda\right\| u_{N}-u\left\|_{b}+\right\| u_{N}-\bar{u}_{N} \|_{b}\right) .\right.
\end{aligned}
$$

Combining with (13), (15), (18) we obtain

$\left\|u-u_{N}\right\|_{a} \leq C(\lambda) C(s)\left(N^{1-s}\|u\|_{s}+N^{-s}\|u\|_{s}\right)$.

This completes the proof.

\section{ITERATED GALERKIN METHOD}

In this section, we shall now introudce iteratedGalerkin method, the iterated Galerkin method method for eigenvalue problems may be dated back to [11]. By using the scheme, the solution of an eigenvalue problem on a big spectral space is reduced to the solution of an eigenvalue problem on a small spectral space and solution of a linear algebraic system on the big spectral space, it provides very accurate approximations with a relatively small number of unknowns, because solving an elliptic eigenvalue problem will not by much more difficult than the solution of some standard elliptic boundary value problem.

Let $\square>N \mathrm{~N}$ and assume that $X_{N} \subset X_{\square}$. We consider the approximation of any eigenvalue $\lambda$ of (2). Here and hereafter we let $\lambda_{N}$ be the eigenvalue of (3) corresponding to $X_{N}$, which satisfies

$$
\left|\lambda_{N}-\lambda\right| \leq C(\lambda) C(s) N^{-s}
$$

Iterated Galerkin method for (2) is constructed as follow

Step1: find $\left(\lambda_{N}, u_{N}\right) \in R \times X_{N}$, such that $\left\|u_{N}\right\|_{b}=1$ and

$$
a\left(u_{N}, v\right)=\lambda_{N} b\left(u_{N}, v\right), \quad \forall v \in X_{N} .
$$

Step 2: find $u_{\square}^{*} \in X_{\square}$, such that

$$
a\left(u_{N}^{*}, v\right)=\lambda_{\square} b\left(u_{\square}, v\right), \quad \forall v \in X_{\square} .
$$

Step 3: compute the Rayleigh quotient

$$
\lambda_{\square}^{*}=\frac{a\left(u_{\square}^{*}, u_{\square}^{*}\right)}{\left(u_{\square}^{*}, u_{\square}^{*}\right)} .
$$

It is seen from Theorem 3.1 that associated with the eigenfunction $u_{N}$ obtained by step 1 , there exists an exact eigenfunction u of (2) satisfying $\left\|u_{N}\right\|_{b}=1$ and

$\left\|u-u_{N}\right\|_{b}+N^{-1}\left\|u-u_{N}\right\|_{a} \leq C(\lambda) C(s) N^{-s}$.

Proposition 4.1 Let $(\lambda, u)$ be an eigenvalue pair of (2).

For any $w \in H_{0}^{1}(\Omega) \backslash\{0\}$,

$\frac{a(w, w)}{b(w, w)}-\lambda=\frac{a(w-u, w-u)}{b(w, w)}-\lambda \frac{b(w-u, w-u)}{b(w, w)}$.

Theorem 4.1 Let $\left(\lambda_{\square}^{*}, u_{\square}^{*}\right)$ be obtained from the iterated Galerkin method. If $X_{N} \subset X_{\square}$, then

$$
\begin{aligned}
& \left\|u_{\square}^{*}-u\right\|_{a} \leq C(\lambda) C(s)\left(\square^{1-s}+N^{-s}\right), \\
& \mid \lambda_{\square}^{*}-\lambda \|_{a} \leq C(\lambda) C(s)\left(\square^{2-2 s}+N^{-2 s}\right) .
\end{aligned}
$$

Proof. Assume $u^{*}$ be an exact solution of (21), combining with (2) we have

$$
a\left(u-u^{*}, v\right)=b\left(\lambda u-\lambda_{N} u_{N}\right), \forall v \in H_{0}^{1}(\Omega) .
$$

Take $v=u-u^{*}$, we obtain

$$
\begin{aligned}
& \left\|u-u^{*}\right\|_{a} \leq C\left(\left\|u_{N}\right\|_{b}\left|\lambda_{N}-\lambda\right|+\lambda\left\|u-u_{N}\right\|_{b}\right) \\
& \leq C(\lambda) C(s) N^{-s} .
\end{aligned}
$$

Because $u_{\square}^{*}$ Nbe an approximate solutions of (21), by (6) we have

$$
\begin{aligned}
& \left\|u_{\square}^{*}-u^{*}\right\|_{a} \leq C(\lambda) C(s) \square{ }^{1-s}\|u\|_{s}, \\
& \left\|u_{\square}^{*}-u^{*}\right\|_{a} \leq C(\lambda) C(s) \square^{1-s}\|u\|_{s} .
\end{aligned}
$$

Using triangle inequality, we obtain

$$
\left\|u_{\square}^{*}-u\right\|_{a} \leq C(\lambda) C(s)\left(\square^{1-s}+N^{-s}\right) .
$$

Takeing $w=u_{\square}^{*}$ in Proposition 4.1, we have

$$
\frac{a\left(u_{\square}^{*}, u_{\square}^{*}\right)}{b\left(u_{\square}^{*}, u_{\square}^{*}\right)}-\lambda=\frac{\left\|u_{\square}^{*}-u\right\|_{a}}{b\left(u_{\square}^{*}, u_{\square}^{*}\right)}-\frac{\left\|u_{\square}^{*}-u\right\|_{b}^{2}}{b\left(u_{\square}^{*}, u_{\square}^{*}\right)} .
$$

Combinig with (22), we obtain

$$
\left|\lambda_{\square}^{*}-\lambda\right| \leq \frac{\left\|u_{\square}^{*}-u\right\|_{a}}{b\left(u_{\square}^{*}, u_{\square}^{*}\right)} .
$$

This completes the proof. 


\section{NUMERICAL EXPERIMENTS}

In this section, we report a simple numerical experiments for a second order elliptic operator. We consider the following eigenvalue problem:

$$
\begin{aligned}
& -u^{\prime \prime}=\lambda u, x \in(-1,1), \\
& u(-1)=u(1)=0 .
\end{aligned}
$$

The eigenvalue of above equation are easily seen to be given by

$$
\lambda_{k}=\frac{k^{2} \pi^{2}}{4}, k=1,2,3, \cdots
$$

with the correspinding normalized eigenfunctions

$$
u(x)=\left\{\begin{array}{l}
\cos \left(\frac{1}{2} k \pi x\right), k=\text { odd }, \\
\sin \left(\frac{1}{2} k \pi x\right), k=\text { even } .
\end{array}\right.
$$

Now we apply the algorithm to solve the problem, these eistimates mean that we can obtain asymptotically optimal errors by taking $\square=O\left(N^{\frac{s}{s-1}}\right)$. If $\lambda$ is the first eigenvalue of the problem, then by Throrem 4.1, we have

$$
\left\|u_{\square}^{*}-u\right\|_{a}=O\left(\square^{1-s}\right),\left|\lambda_{\square}^{*}-\lambda\right|=O\left(\square^{2-2 s}\right) .
$$

The results shown in Table 1 are consistent with the above estimates, this numerical experiments are carried out to verify the theoretical prediction.

Table 1. Iterated Galerkin method for elliptic eigenvalue problems

\begin{tabular}{|c|c|c|c|c|}
\hline$N$ & $\lambda_{N}$ & $\lambda_{N}^{*}$ & $\left|\lambda_{N}-\lambda\right|$ & $\left|\lambda_{N}^{*}-\lambda\right|$ \\
\hline 3 & 2.5 & 2.46774193548387 & $3.25989 \times 10^{-2}$ & $3.40899 \times 10^{-4}$ \\
\hline 4 & 2.4674374053292 & 2.46740121355576 & $3.63050 \times 10^{-5}$ & $1.13283 \times 10^{-7}$ \\
\hline 6 & 2.4674011087466 & 2.46740110027234 & $8.47426 \times 10^{-9}$ & $6.85050 \times 10^{-12}$ \\
\hline 8 & 2.46740110027299 & 2.46740110027234 & $6.50501 \times 10^{-13}$ & $3.51632 \times 10^{-16}$ \\
\hline 10 & 2.467401100272340 & 2.46740110027233 & $8.88178 \times 10^{-16}$ & $1.56635 \times 10^{-16}$ \\
\hline 12 & 2.467401100272339 & 2.467401100272339 & $4.44089 \times 10^{-16}$ & $1.56632 \times 10^{-16}$ \\
\hline 14 & 2.467401100272340 & 2.467401100272339 & $8.88178 \times 10^{-16}$ & $1.56632 \times 10^{-16}$ \\
\hline 16 & 2.467401100272339 & 2.467401100272339 & $4.44089 \times 10^{-16}$ & $1.56632 \times 10^{-16}$ \\
\hline
\end{tabular}

\section{REFERENCES}

[1] I. Babuska, J. Osborn, Eigenvalue problems. Handbook of numerical analysis, Elsevier Science Publishers 1991, 2: 641.

[2] C. Bernardi, G. Coppoletta, Y. Maday, "Some spectral approximations of two-dimensional fourth-order problems," Mathematics of computation, 1992, 59(199): 63-76.

[3] C. Canuto, M. Y. Hussaini, A. Quarteroni, et al., Spectral methods in fluid dynamics, Springer-Verlag, 1988.

[4] O. Axelsson, W. Layton, "A two-level discretization of nonlinear boundary value problems," SIAM journal on numerical analysis,1996, 33(6): 2359-2374.

[5] C. N. Dawson, M. F. Wheeler, "Two-grid methods for mixed finite element approximations of nonlinear parabolic equations," Contemporary Mathematics, 1994, 180: 191-191

[6] J. Xu, "Two-grid discretization techniques for linear and nonlinear PDEs," SIAM Journal on Numerical Analysis, 1996, 33(5): 17591777.

[7] M. Marion, J. Xu, "Error estimates on a new nonlinear Galerkin method based on two-grid finite elements," SIAM Journal on Numerical Analysis, 1995, 32(4): 1170-1184.

[8] W. Layton, W. Lenferink, "Two-level Picard and modified Picard methods for the Navier-Stokes equations," Applied Mathematics and Computation, 1995, 69(2): 263-274.

[9] T. Utnes. "Two - grid finite element formulations of the incompressible Navier-Stokes equations," Communications in numerical methods in engineering, 1997, 13(8): 675-684.

[10] J. Shen, "Efficient spectral-Galerkin method I. Direct solvers of second-and fourth-order equations using Legendre polynomials," SIAM Journal on Scientific Computing, 1994,

[11] Q. Lin, G. Xie, "Accelerating the finite element method in eigenvalue problems," Ke Xue Tong bao, 1981, 26, 449-452(in Chinese). 